\title{
MRI Abnormalities Predominate in the Bottom Part of the Sulcus with Type II Focal Cortical Dysplasia: A Quantitative Study
}

\author{
(D) Z. Liu, (D)W. Hu, (D) Z. Sun, (D)X. Wang, (D). Liu, (D)X. Shao, (D). Zhang, (D) Y. Ma, and (D). Zhang
}

\begin{abstract}
BACKGROUND AND PURPOSE: Type II focal cortical dysplasia is a common histopathological substrate in focal epilepsy. This study explored the spatial distribution of abnormal findings on MR imaging across the sulcus with type II focal cortical dysplasia using quantitative MR imaging postprocessing techniques.
\end{abstract}

MATERIALS AND METHODS: The morphometric analysis program and normalized FLAIR signal intensity analysis were applied to retrospectively analyze the MR imaging data of 58 patients with histopathologically confirmed type II focal cortical dysplasia. We divided the dysplastic sulcus into the bottom and nonbottom parts. Then spatial distribution types 1, 2, and 3 were arbitrarily defined as the abnormal findings on MR imaging ( $z$-value $>$ threshold) located in the bottom part, both the bottom and nonbottom parts, and the nonbottom part, respectively. For type 2 , the mean $z$-values and standardized volumes of abnormal findings on MR imaging were compared between the bottom and nonbottom parts.

RESULTS: Abnormal findings on MR imaging were detected by quantitative techniques in 42 of 58 enrolled patients. Among these 42 patients, 38 and 26 patients showed gray-white matter junction blurring and cortical FLAIR hyperintensity, respectively, which were the 2 most common abnormal MR imaging features. Gray-white matter junction blurring manifested as types 1, 2, and 3 in 24, 13, and 1 patient, respectively, and the corresponding counts for cortical FLAIR hyperintensity were 12, 13, and 1 patient. For the 2 most common abnormal findings on MR imaging spatially manifested as type 2, higher mean $z$-values and larger corresponding standardized volumes of abnormalities were found in the bottom part.

CONCLUSIONS: Abnormal findings on MR imaging predominate in the bottom part of the sulcus with type II focal cortical dysplasia, which indicates that this malformation is bottom-of-sulcus-rooted.

ABBREVIATIONS: $\mathrm{FCD}=$ focal cortical dysplasia; $\mathrm{MAP}=$ morphometric analysis program; $\mathrm{nFSI}=$ normalized FLAIR signal intensity

$\mathrm{T}$ ype II focal cortical dysplasia (FCD) is a highly epileptogenic lesion associated with pharmacoresistant epilepsy, and it is characterized by dysmorphic neurons and balloon cells. ${ }^{1}$ FCD type II was first described by Taylor et al, in 1971, ${ }^{2}$ and increasing reports have indicated that it is a common histopathologic substrate in epilepsy surgery. ${ }^{3-6}$ Clinical characteristics, including se-

Received August 14, 2018; accepted after revision October 30.

From the Departments of Neurosurgery (Z.L., X.W., K.Z., J.Z.) and Neurology (X.S.), Beijing Tiantan Hospital, Capital Medical University, Beijing, China; Beijing Neurosurgical Institute (W.H., J.Z.), Capital Medical University, Beijing, China; Department of Neurosurgery (Z.S.), Beijing Tsinghua Changgung Hospital, School of Clinical Medicine, Tsinghua University, Beijing, China; and Department of Pathology (L.L.) and Neurosurgery (Y.M.), Beijing Fengtai Hospital, Beijing, China.

This work is partly supported by grants from the Capital (China) Health Research and Development of Special Fund (2016-1-1071), the Beijing Municipal Science \& Technology Commission (Z161100000216130), the Beijing Municipal Administration of Hospitals' Ascent Plan (DFL20150503), and the Capital Medical University Basic and Clinical Cooperative Research Project (17JL05).

Zhifeng Liu and Wenhan Hu contributed equally to this work. vere partial epilepsy beginning in childhood, stereotyped seizures, high seizure frequency, and extratemporal location, have been associated with this specific malformation. ${ }^{6}$ Electrophysiologic studies have also revealed that repetitive subcontinuous spikes, spikes and waves, polyspikes, or bursts of fast rhythms are reliable biomarkers of FCD type II. ${ }^{7}$

In addition to the above-mentioned electroclinical features, neuroimaging studies have demonstrated MR imaging characteristics of FCD type II, including gray-white matter junction blurring, increased cortical FLAIR signal, abnormal gyration/sulcation, increased cortical thickness, and the transmantle sign. ${ }^{8-10}$ However, most previous studies were based on conventional vi-

Please address correspondence to Jianguo Zhang, MD, PhD, Beijing Neurosurgical Institute, Capital Medical University, Tiantan xili 6, Beijing, 100050, China; e-mail: zjguo73@126.com

- Indicates open access to non-subscribers at www.ajnr.org

http://dx.doi.org/10.3174/ajnr.A5919 
sual analysis, which strongly depends on the training and experience of the interpreting rater and has limited ability to recognize the existence and delineate the extent of FCD lesions. With the development of neuroimaging and computer technology, a large number of image-postprocessing methods have been developed to detect the abnormal findings on MR imaging of FCD quantitatively. ${ }^{11-15}$ In a morphometric analysis program (MAP) first introduced by Huppertz et al, ${ }^{14} 3$ feature images (ie, junction, extension, and thickness images) are derived from a statistical parametric mapping-based algorithm, which was designed to measure the severity of gray-white matter junction blurring, abnormal gyration/sulcation, and cortical thickening, respectively. ${ }^{14,16}$ Normalized FLAIR signal intensity (nFSI) analysis compares individual MR imaging data with a healthy control data base to detect changes in FLAIR signal intensity. ${ }^{12}$ These 2 quantitative methods have high degrees of sensitivity in FCD detection compared with conventional visual inspection. ${ }^{12,13,17}$

Besson et $\mathrm{al}^{18}$ used techniques of automated sulcal extraction and morphometry to explore the spatial relationship between FCD lesions and brain sulci, and they concluded that small FCDs were located at the bottom of a deep sulcus. However, only small lesions were investigated; large FCD lesions with abnormal findings on MR imaging continuously extending to the wall or crown of the sulci remained unexplored in their series. The present study aimed to describe the patterns of the distribution of abnormal findings on MR imaging across the type II dysplastic sulci using quantitative techniques. We believe our work can provide further helpful information to better understand the mechanism of FCD pathogenesis as well as principles of intracranial electrode implantation and resective surgery planning.

\section{MATERIALS AND METHODS \\ Patients}

We retrospectively included patients who underwent a resective epilepsy operation in Beijing Tiantan-Fengtai Epilepsy Center from January 2015 to December 2017 using the following criteria: 1) The histopathologic finding was type II FCD, according to the 2011 International League Against Epilepsy FCD classification system. FCD type II was defined as abnormal cortical lamination with dysmorphic neurons alone (IIa) or together with balloon cells (IIb). ${ }^{1}$ 2) Presurgical3D T1 and FLAIR images were available. Patients with low-quality MR images due to noise or movement artifacts were excluded. 3) The patient was older than 5 years of age, ${ }^{15,19}$ and 4) no cranial surgery had been performed previously.

This research was approved by the institutional review board of the Beijing Tiantan Hospital (approval number KY2017-043-02), and informed consent was obtained from all included participants.

\section{MR Imaging Acquisition}

The MR images were obtained on a 3T Verio scanner (Siemens, Erlangen, Germany), including 3D T1 sagittal MPRAGE (TR/TE = $1900 / 2.53 \mathrm{~ms}, \mathrm{TI}=900 \mathrm{~ms}$, matrix $=256 \times 256$, thickness $=1.0$ $\mathrm{mm}), \mathrm{T} 2$ axial $(\mathrm{TR} / \mathrm{TE}=7030 / 110 \mathrm{~ms}$, matrix $=256 \times 320$, thickness $=3 \mathrm{~mm})$, FLAIR axial $(\mathrm{TR} / \mathrm{TE}=8000 / 94 \mathrm{~ms}, \mathrm{TI}=2371.5 \mathrm{~ms}$, matrix $=424 \times 512$, thickness $=3 \mathrm{~mm})$, FLAIR sagittal $(\mathrm{TR} / \mathrm{TE}=$ $8000 / 96 \mathrm{~ms}, \mathrm{TI}=2371.2 \mathrm{~ms}$, matrix $=236 \times 256$, thickness $=3$ $\mathrm{mm})$, and FLAIR coronal $(\mathrm{TR} / \mathrm{TE}=8000 / 96 \mathrm{~ms}, \mathrm{TI}=2371.2 \mathrm{~ms}$, matrix $=408 \times 512$, thickness $=3 \mathrm{~mm}$ ) sequences.

\section{Visual Inspection}

Two epileptologists (X.S., W.H.) performed conventional visual analysis of the MR images independently, and discrepancies were resolved through discussion. The 2 reviewers defined positive findings on MR imaging when FCD-associated abnormal findings were identified during this procedure.

\section{MR Imaging Postprocessing and Dysplastic Sulci Labeling}

Two SPM8 (http://www.fil.ion.ucl.ac.uk/spm/software/spm8) postprocessing algorithms, MAP and nFSI, were used to quantify the abnormal findings on MR imaging. Extension, junction, and thickness images composed of $z$-values were derived from MAP by comparing individual MR imaging data with a healthy control data base. These images are used to measure the severity of abnormal gyration/ sulcation, gray-white matter junction blurring, and cortical thickening, respectively. ${ }^{16}$ Gray-white matter junction blurring may lead to inaccurate segmentation of gray or white matter probability images, which can result in incorrect calculation of cortical thickness. To avoid the cortical pseudothickening induced by gray-white matter junction blurring, we adjusted the gray matter probability image segmented for cortical thickness calculation by subtracting the binary image (derived from the 3D T1 image in the workflow of junction image calculation). FLAIR intensity changes in gray and white (transmantle sign) matter were quantitatively measured by nFSI, resulting in a $z$-value-based image. An epileptologist (K.Z.) blinded to the conventional visual results used $z$ score thresholds of 6 (extension image), 3.5 (junction image), 4 (thickness image), or 3 (nFSI image) to identify candidate postprocessing positive (postprocessing + ) regions. ${ }^{12,15,19,20}$ The coregistered structural MR imaging and postsurgical MR imaging or CT were visually inspected at the candidate postprocessing + region. The patient was classified as postprocessing + if the candidate region was considered abnormal on the structural scan and was removed according to the postsurgical MR imaging or CT. The details of image-processing steps and threshold selection were described in previous articles. ${ }^{12,15-17,19,20}$ The dysplastic sulcus with abnormal findings on MR imaging was manually labeled in the 3D T1 MR image according to the postprocessing results, and the corresponding ROI was drawn using VOI tools of MRIcron (http://people.cas.sc.edu/rorden/mricron/index.html) (Fig 1).

\section{Division of the Dysplastic Sulcus}

There is no previous literature describing the extent of the bottom part of a sulcus. We first delineated this extent according to our knowledge. On the basis of the structural MR image and corresponding ROI image, a reviewer (Z.L.) blinded to the postprocessing results drew a line intersecting the bottom point of the sulcus and parallel to the tangent to the interior border of the gray matter. This line divided the ROI of the dysplastic sulcus into the bottom and nonbottom parts (Fig 2A).

\section{Patterns of Distribution of Abnormal Findings on MR Imaging}

We defined spatial distribution types 1,2, and 3 as abnormal findings on MR imaging ( $z$-value $>$ corresponding threshold) 


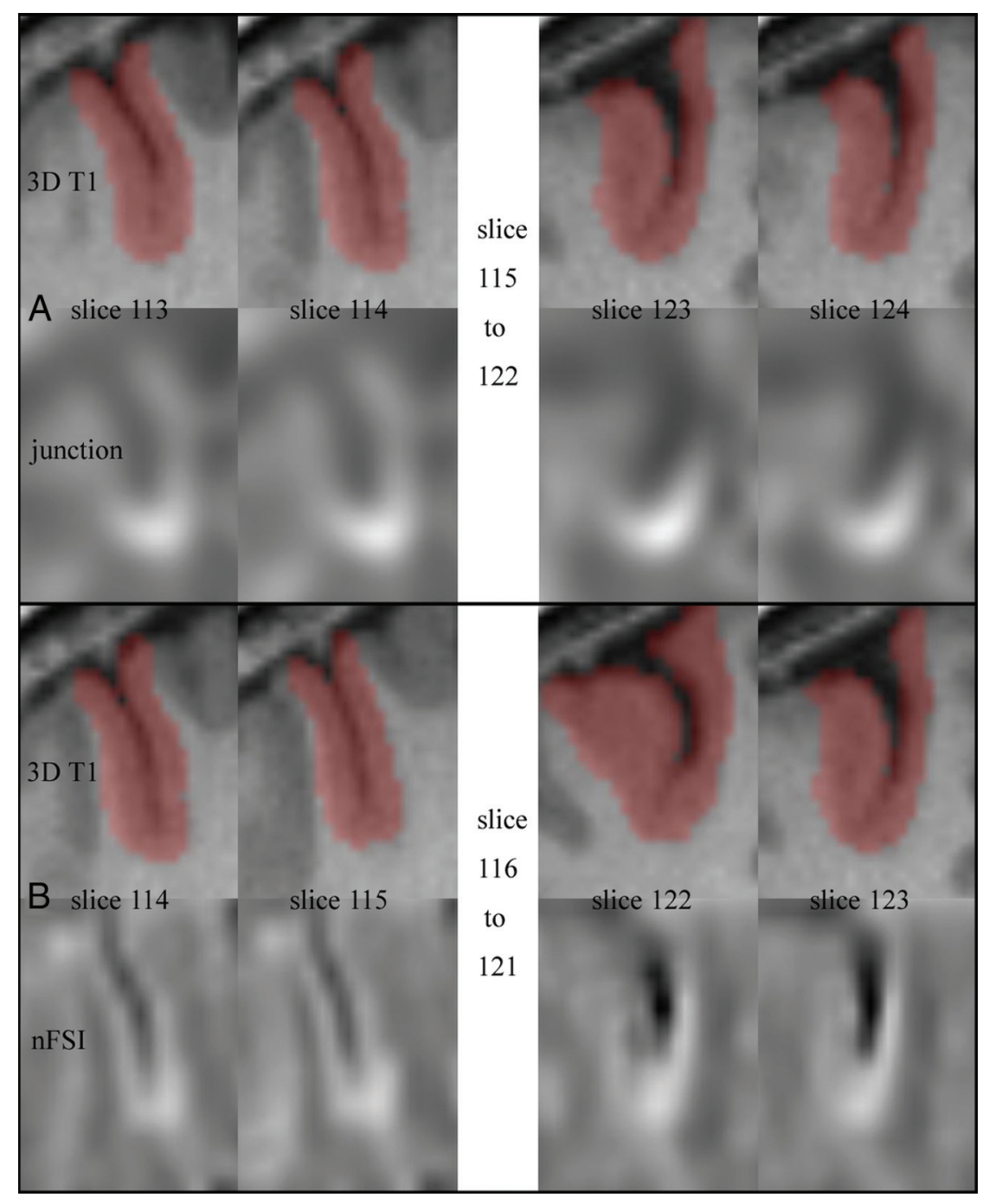

FIG 1. Dysplastic sulcus labeling. A, Dysplastic sulcus with an abnormality of gray-white matter junction blurring (coronal). B, Dysplastic sulcus with an abnormality of cortical FLAIR hyperintensity (coronal).

located in the bottom part, both the bottom and nonbottom parts, and the nonbottom part, respectively (Fig $2 B-D$ ).

\section{Comparisons between the Bottom and Nonbottom Parts}

For patients with type 2 distribution, we further compared the severities of abnormal findings on MR imaging between the bottom and nonbottom parts. The corresponding postprocessing images were multiplied by the mask images of the bottom or nonbottom part to obtain the statistical images of the 2 parts. The mean $z$-value in the bottom or nonbottom part of each statistical image was calculated. Moreover, the number of voxels with a $z$-value more than the previously defined threshold in the bottom or nonbottom part was divided by the total number of voxels of the corresponding part to obtain the standardized volume of the abnormality. All calculations during this step were performed in the platform of Matlab R2013a (MathWorks, Natick, Massachusetts). The mean $z$-values and standardized volumes of abnormalities between the bottom and nonbottom parts were compared in patients with type 2 distribution.

\section{Postoperative Outcome}

The Engel Scale was used to rate postoperative outcome. Seizure freedom was strictly defined as Engel Ia (seizure-free, no auras). ${ }^{21}$

\section{Statistical Analyses}

The Fisher exact test (2-tailed) was performed for statistical comparisons of independent categoric data. The independent $t$ test (2-tailed) was performed for comparisons of independent numeric data if the variables were normally distributed; otherwise, the Mann-Whitney $U$ test was used. Significance was defined as $P \leq .05$. The statistical tests were performed with SPSS 22.0 (IBM, Armonk, New York).

\section{RESULTS \\ General Information}

Between January 2015 and December 2017, four hundred seventy-one patients underwent resective surgery for refractory focal epilepsy at Beijing Tiantan-Fengtai Epilepsy Center. Among them, 72 patients had the histopathologic diagnosis of FCD II. One, 9, and 4 patients were excluded due to obvious artifacts on MR images, age younger than 5 years, and previous cranial operations, respectively. Fiftyeight patients (23 female and 35 male) were ultimately included in this study. The mean age at the operation was $18.78 \pm 9.59$ years, and the mean epilepsy duration was $7.89 \pm 5.37$ years. Thirty-seven patients underwent stereoelectroencephalography monitoring before resective surgery. The FCD lesions were located in the frontal lobe in 38 (65.52\%) patients, parietal lobe in $8(13.79 \%)$ patients, temporal lobe in $4(6.90 \%)$ patients, occipital lobe in $4(6.90 \%)$ patients, insular lobe in $3(5.17 \%)$ patients, and multiple lobes in $1(1.72 \%)$ patient. At a mean postoperative follow-up of $23.81 \pm 9.60$ months, 50 patients $(86.2 \%)$ became seizure-free (Engel Ia).

\section{Detection Rates of Conventional Visual Analysis and Image Postprocessing Assistant Analysis}

In general, 27 (46.55\%) FCD lesions were detected by visual inspection. In addition to the 27 lesions, another 15 (25.86\%) subtle FCDs were detected under the assistance of image postprocessing techniques, leading to the detection rate of $72.41 \%$. The findings of remaining 16 (27.59\%) FCDs were strictly negative on MR imaging and were detected by interictal PET with $\left[{ }^{18} \mathrm{~F}\right]$ FDG or stereoelectroencephalography (Fig 3).

\section{Frequencies of Abnormal Findings on MR Imaging}

As shown in the Table, among the 42 FCD lesions with detectable abnormal findings on MR imaging, gray-white matter junction 


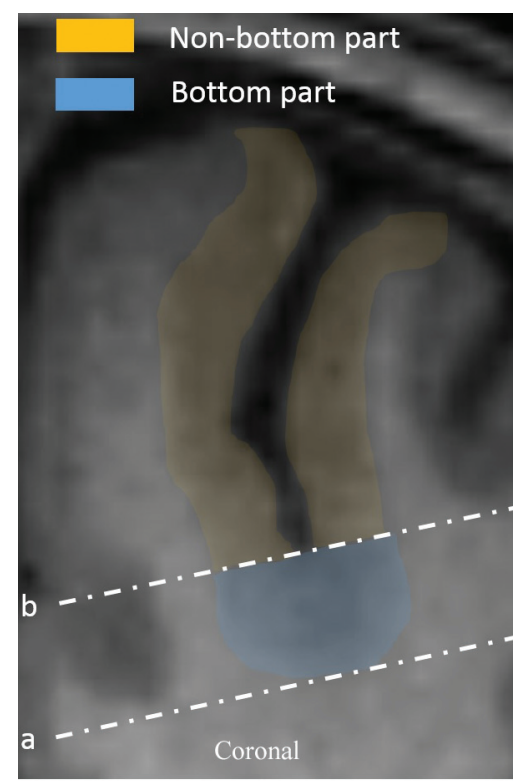

A

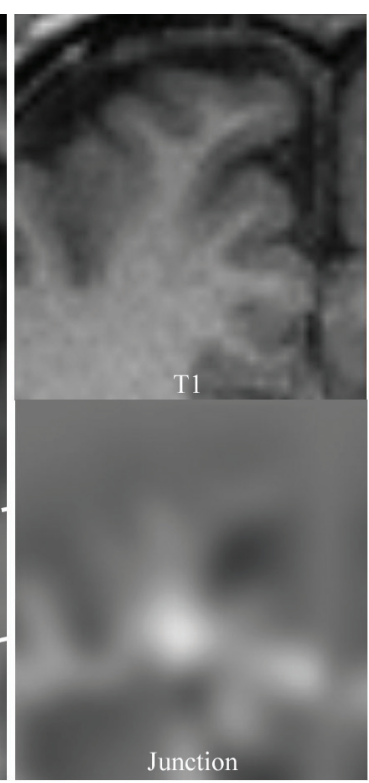

B

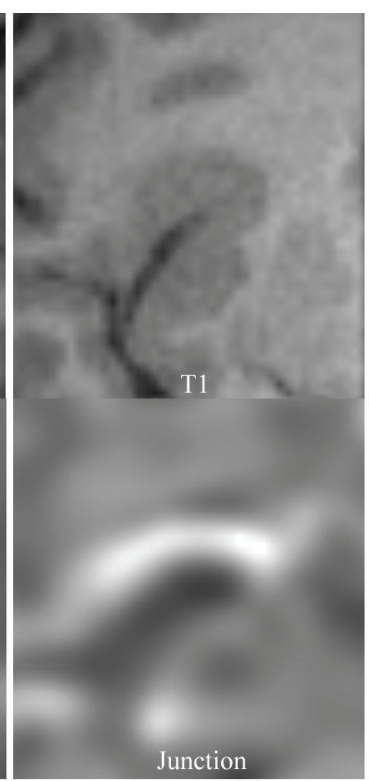

C

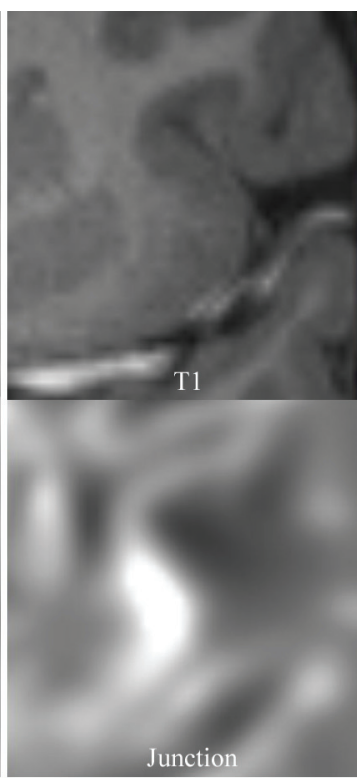

D

FIG 2. Schematic diagram of the divisions of the sulcus and the types of spatial distribution of abnormal findings on MR imaging. A, Line $a$ is tangential to the interior border of the gray matter in the sulcus bottom, and line $b$ is parallel to line $a$, intersecting the bottom point of the sulcus. Gray matter interior and exterior to line $b$ is defined as the bottom and the nonbottom parts, respectively. If one considered gray-white matter junction blurring, for example, spatial distribution types $1(B), 2(C)$, and $3(D)$ are defined as the abnormalities located in the bottom part, both the bottom and nonbottom parts, and the nonbottom part, respectively.

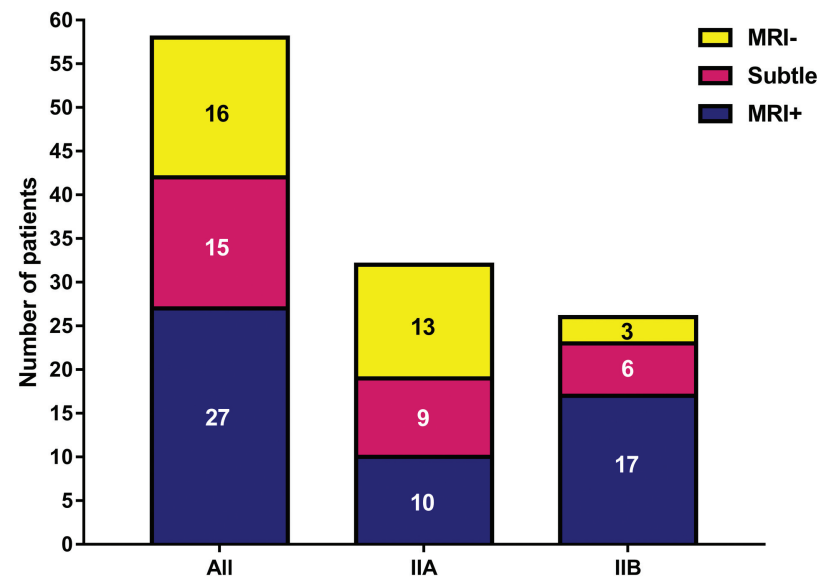

FIG 3. Abnormal findings on MR imaging detected by conventional visual analysis and postprocessing techniques. MRI+ indicates positive by conventional visual analysis; Subtle, negative by conventional visual analysis but positive by postprocessing; MRI-, negative by postprocessing.

blurring was the most common $(n=38)$, followed by cortical FLAIR hyperintensity $(n=26)$, the transmantle sign $(n=13)$, abnormal gyration or sulcation $(n=7)$, and cortical thickening $(n=4)$. Type IIb lesions significantly more often showed detectable abnormalities $(P=.015)$, including gray-white matter junction blurring $(P=.029)$ and cortical FLAIR hyperintensity $(P=$ $.001)$, compared with type IIa lesions; the transmantle sign was an exclusive feature of FCD IIb $(P<.001)$.

\section{Patterns of Distribution of Abnormal Findings on MR Imaging}

The abnormalities of gray-white matter junction blurring located in the bottom part (type 1) of dysplastic sulci in 24 patients (IIa, $n=10 ; \mathrm{IIb}, n=14)$, consecutively extended to the wall or crown (type 2 ) in 13 patients (IIa, $n=6$; IIb, $n=7$ ) and were located in the nonbottom part (type 3 ) in 1 patient with IIa. For the abnormality of cortical FLAIR hyperintensity, 12 (IIa, $n=2$; IIb, $n=$ 10), 13 (IIa, $n=6$; IIb, $n=7$ ), and 1 (IIb, $n=1$ ) lesion showed type 1, 2, and 3 spatial distribution, respectively (Fig 4). All abnormalities of the transmantle sign and abnormal gyration/sulcation showed a type 1 distribution. Regarding cortical thickening, 2 lesions showed a type 2 distribution and the other 2 showed a type 3 distribution.

\section{Comparisons between the Bottom and Nonbottom Parts}

Because all abnormalities of the transmantle sign and abnormal gyration/sulcation were located in the bottom part and cases with cortical thickening were rare in our cohort, only $z$-values of the junction and nFSI images were taken into account for comparisons. In patients with gray-white matter junction blurring consecutively extending from the bottom to the wall or crown (type 2 distribution), the mean $z$-value in the bottom part was higher than that in the nonbottom part (all: $3.91 \pm 1.21$ versus $0.43 \pm 0.60, P<.001$; subgroup of FCD IIa: $4.37 \pm 1.41$ versus $0.41 \pm 0.41, P<.001$; subgroup of FCD IIb: $3.51 \pm 0.94$ versus $0.44 \pm 0.77, P<.001$ ), and the standardized volume of abnormalities was also larger in the bottom part (all: $0.56 \pm 0.19$ versus $0.09 \pm 0.06, P<.001$; subgroup of FCD IIa: $0.60 \pm 0.18$ versus $0.09 \pm 0.04, P<.001$; subgroup of FCD IIb: $0.52 \pm 0.20$ versus $0.08 \pm 0.08, P<.001)$. Regarding FLAIR signal changes, the mean $z$-value was higher (all: $2.52 \pm 0.63$ versus $0.92 \pm$ $0.50, P<.001$; subgroup of FCD IIa: $2.39 \pm 0.87$ versus $0.80 \pm 0.24$, $P<.001$; subgroup of FCD IIb: $2.63 \pm 0.37$ versus $1.03 \pm 0.65, P<$ .001 ) and the standardized volume of abnormalities was larger (all: $0.34 \pm 0.18$ versus $0.06 \pm 0.05, P<.001$; subgroup of FCD IIa: $0.28 \pm 0.22$ versus $0.04 \pm 0.04, P<.001$; subgroup of FCD IIb: 
Frequencies of abnormal findings on MRI measured by quantitative methods

\begin{tabular}{lclcc}
\hline & All $(\boldsymbol{n}=\mathbf{5 8})$ & Ila $(\boldsymbol{n}=\mathbf{3 2})$ & Ilb $(\boldsymbol{n}=\mathbf{2 6})$ & $\boldsymbol{P}$ (Ila vs IIb) \\
\hline Gray-white matter junction blurring & $38(65.52 \%)$ & $17(53.13 \%)$ & $21(80.77 \%)$ & .029 \\
Cortical FLAIR hyperintensity & $26(44.83 \%)$ & $8(25 \%)$ & $18(69.23 \%)$ & .001 \\
Cortical thickening & $4(6.90 \%)$ & $2(6.25 \%)$ & $2(7.69 \%)$ & $>.999$ \\
Abnormal gyrus or sulcus & $7(12.07 \%)$ & $5(15.63 \%)$ & $2(7.69 \%)$ & .614 \\
Transmantle sign & $13(22.41 \%)$ & $0(0 \%)$ & $13(100 \%)$ & $<.001$ \\
Normal findings & $16(27.59 \%)$ & $13(40.63 \%)$ & $3(11.54 \%)$ & .015 \\
\hline
\end{tabular}
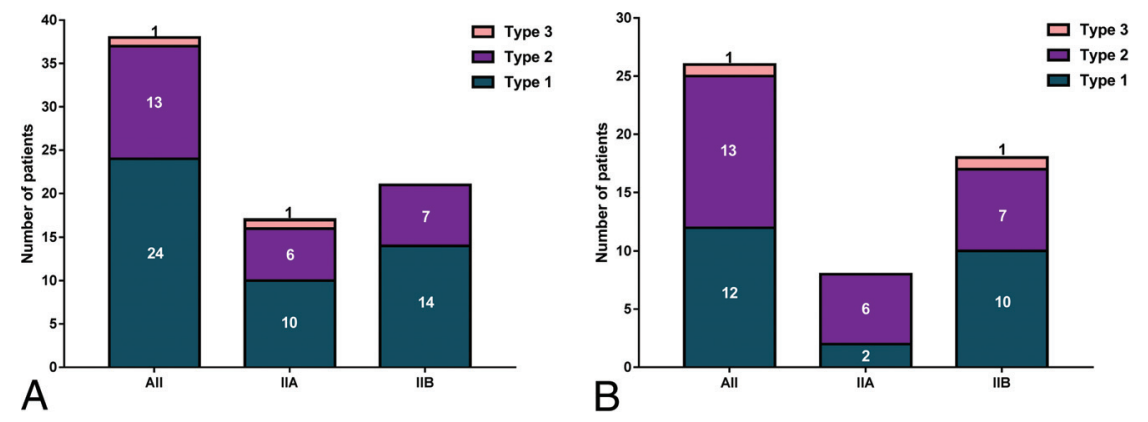

FIG 4. Statistical data of the 3 spatial distribution types of abnormalities on MR imaging. $A$, Gray-white matter junction blurring. B, Cortical FLAIR hyperintensity.

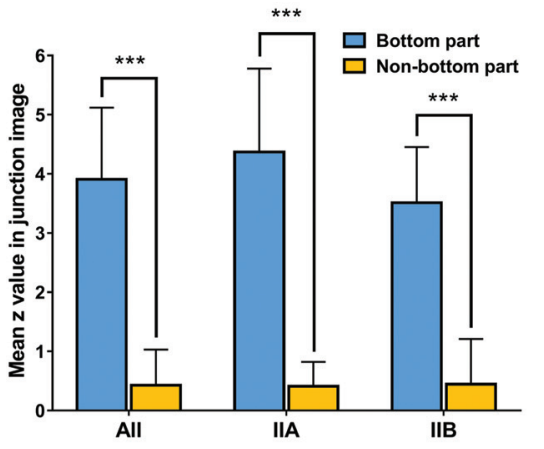

A

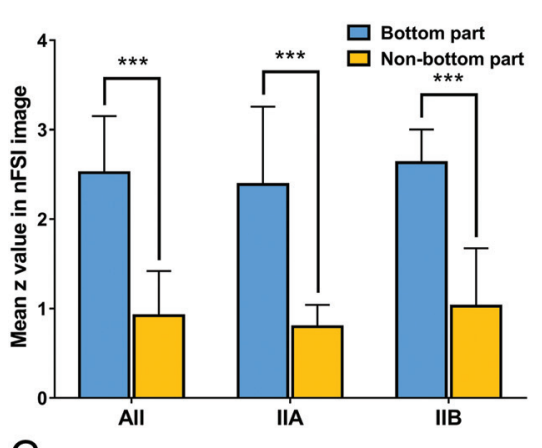

C

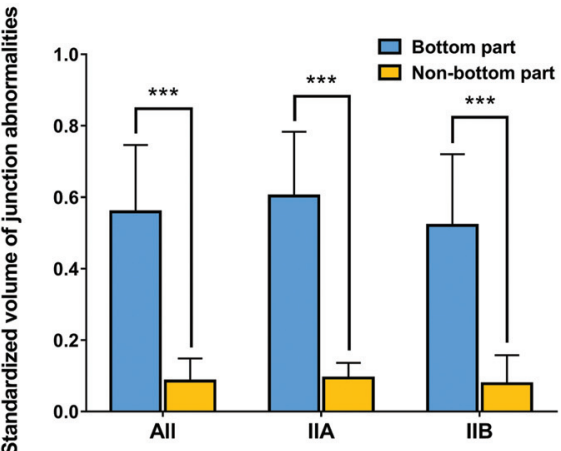

$\mathrm{B}$

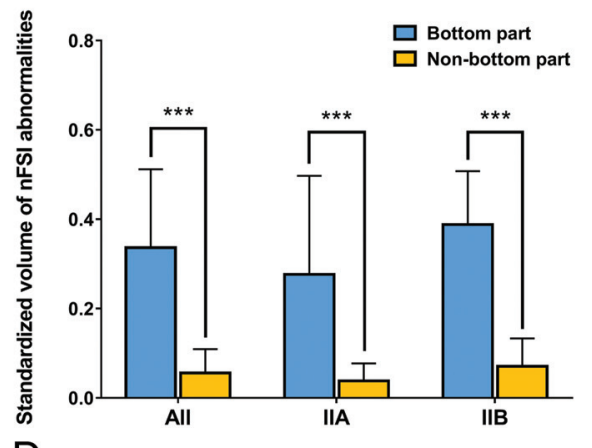

FIG 5. Comparisons between the bottom and nonbottom parts in patients with a type 2 abnormality distribution. $A$, Comparison of $z$-values derived from the junction image (designed to measure gray-white matter junction blurring) of MAP. B, Comparison of standardized volumes of abnormalities $(z>3.5)$ in the junction image. $C$, Comparison of $z$-values derived from the $\mathrm{nFSI}$ image (designed to measure cortical FLAIR hyperintensity). D, Comparison of standardized volumes of abnormalities $(z>3)$ in the $\mathrm{nFSI}$ image.

$0.39 \pm 0.12$ versus $0.07 \pm 0.06, P<.001)$ in the bottom part compared with that in the nonbottom part (Fig 5 ).

\section{DISCUSSION}

Knowledge of the MR imaging features is crucial for recognition and detection of FCD II lesions, and understanding the spatial distribution of abnormal findings on MR imaging is important for deep electrode implantation and lesion resection. The present retrospective study quantitatively revealed that gray-white matter junction blurring and cortical FLAIR hyperintensity were the 2 most common abnormal findings on MR imaging of type II FCD, and the transmantle sign was exclusive to FCD IIb. Moreover, abnormal findings on MR imaging predominated in the bottom part of the sulcus with type II FCD.

Bottom-of-sulcus dysplasia was first proposed by the 2005 revision of the Barkovich classification. It was defined as a subtype of FCD with balloon cells (FCD IIb). ${ }^{22}$ FCD IIa was excluded because it had different imaging features, different outcomes after the seizure operation, and clearly different etiologies. ${ }^{4,5,22-24}$ In 2008, a study by Besson et $\mathrm{al}^{18}$ indicated that small FCD lesions were located at the bottom of a deep sulcus. The present study demonstrates that in addition to FCD IIb, the abnormal findings on MR imaging ( $n=10$ for gray-white matter junction blurring and $n=2$ for cortical FLAIR hyperintensity) of FCD IIa lesions could also be located in the bottom part of the sulcus. From the perspective of spatial location, these FCD IIa lesions should not be excluded from bottom-of-sulcus dysplasia. The exclusion of FCD IIa in the classification by Barkovich is most likely explained by the use of low-field-strength or low-resolution MR images. Small FCD IIa lesions located in the bottom part of the sulcus, frequently manifesting as subtle gray-white matter junction blurring and lacking notable characteristics such as the transmantle sign, tend to be overlooked during conventional visual analysis. As a consequence of negative findings on MR imaging, it is difficult to localize those lesions and the seizure outcomes are poor. ${ }^{25,26}$

In addition to subtle lesions located at the bottom, other larger lesions with abnormalities continuously extending from the bottom to the wall were also analyzed in our study. Among these lesions, quantitative analyses indicated that abnormal findings on MR imaging predominated in the bottom part of the sulcus. Based on the above-mentioned findings, we postulated that the bottom of the sulcus was the "root" of type II FCD lesions and proposed the concept of 
bottom-of-sulcus rooted dysplasia. The lesions with type 1 and 2 spatial distributions were all bottom-of-sulcus-rooted dysplasia. The only difference between them we could see was the volume of the lesions.

The mechanism of cortical folding is unclear. Two hypotheses have dominated views on this topic. The first is the intracortical differential growth hypothesis, whereby the differential expansion of upper-versus-lower neuronal layers causes the surface to buckle or fold. ${ }^{27}$ The cortex is thicker at sites of growing gyri and thinner beneath developing sulci. ${ }^{28}$ The cortex forms its 6-layered structure until gestational week 18 , before cortical folding. We postulate that the sites where FCD lesions are located, being the weak points of tangential tension due to the disruption of cortical layers, tend to be folded into the sulci. The second is the axonal tension hypothesis, which posits that tension generated by axons drives cortical folding by pulling strongly interconnected regions together. ${ }^{29}$ According to this theory, gyri are bulked between areas with strong axonal connections as axons draw them together, and sulci are folded between areas weakly connected or even without links. Decreased subcortical fiber connectivity in FCD has been demonstrated, ${ }^{30-32}$ which may be the main reason that this kind of lesion is bottom-of-sulcus-rooted.

Our study also provided a rule for interpreting the results of postprocessing images in the detection of FCD lesions. The sample cases of FCD II presented in the literature of image postprocessing showed that most dysplastic areas highlighted by the extension, junction, and nFSI images were located in the inferior parts of the dysplastic sulci. ${ }^{12-14}$ The results of the present study are consistent with the findings from previous publications. Huppertz, ${ }^{16}$ the introducer of MAP, claimed that this method does not detect the lesion automatically, so a visual confirmation at the MAP-highlighted area on coregistered structural MR imaging was still necessary. The interpretation of the postprocessing images requires some experience. Moreover, false-positives exist because the postprocessing images may highlight areas that have no pathologic correlates in the structural MR images. ${ }^{16}$ If the electroclinical data indicate an FCD II lesion, areas at the crown of the gyri highlighted by any postprocessing images need extremely careful inspection because this kind of lesion is bottom-of-sulcus-rooted.

Subdural and depth electrodes are widely used in localizing epileptogenic zones. Although subdural electrodes have advantages, including large cortical coverage and convenience in cortical mapping, ${ }^{33}$ contacts of subdural electrodes, which are placed on the surface of the brain, cannot directly record the signals of FCD deeply buried in the sulcus. Thus, depth electrodes are preferred in localizing FCD II during the phase 2 evaluation. To go close to the FCD lesion rooted at the bottom of the sulcus, the trajectory of the depth electrode should run through the inferior portion of the dysplastic sulcus. For lesion resection, the depth of resection of the sulcus directly affects the postsurgical seizure outcome. In other words, the gray matter at the bottom should be completely removed to reveal the underlying white matter, especially for the sulcus, where vessels pass at the bottom.

The main limitation of the present study is the exclusion of pediatric patients younger than 5 years of age due to the absence of healthy controls with a similar age distribution. Younger brains show significant structural differences compared with adult brains, and bias might have occurred during the unequal comparisons. However, the prevalence rate of FCD II in these pediatric patients undergoing an epilepsy operation was high. Another limitation is that some cases with electroclinical or neuroimaging data strongly indicating FCD II were not included due to negative histopathologic findings. The main cause, we postulate, is the fragmentation of specimens during the resective surgery. Selection bias might have been induced by the above-mentioned limitations in our study.

\section{CONCLUSIONS}

We explored the spatial distribution of abnormal findings on MR imaging across the sulci containing FCD II lesions using quantitative techniques. Our results suggest that gray-white matter junction blurring and cortical FLAIR hyperintensity are the 2 most common characteristics, which predominate in the bottom part of the sulcus with type II FCD. The findings of our study indicate that FCD II is bottom-of-sulcus-rooted and provide helpful information for postprocessing image interpretation, intracranial electrode implantation, and resection of this kind of lesion.

Disclosures: Kai Zhang—RELATED: Grant: Capital Medical University Beijing, Comments: Capital Medical University Basic and Clinical Cooperative Research Project (17)L05).* Jianguo Zhang—RELATED: Grant: Beijing Municipal Commission of Health and Family Planning, Beijing Municipal Science \& Technology Commission, Beijing Municipal Administration of Hospitals, Comments: Capital (China) Health Research and Development of Special Fund (2016-1-1071), the Beijing Municipal Science \& Technology Commission (Z161100000216130), Beijing Municipal Administration of Hospitals' Ascent Plan (DFL20150503)*; Consulting Fee or Honorarium: Beijing Municipal Administration of Hospitals, Comments: Beijing Municipal Administration of Hospitals' Ascent Plan (DFL20150503)*; Support for Travel to Meetings for the Study or Other Purposes: Beijing Municipal Commission of Health and Family Planning, Comments: Capital (China) Health Research and Development of Special Fund (2016-11071)*; Fees for Participation in Review Activities such as Data Monitoring Boards, Statistical Analysis, Endpoint Committees, and the Like: Beijing Municipal Administration of Hospitals, Comments: Beijing Municipal Administration of Hospitals' Ascent Plan (DFL20150503). * *Money paid to the institution.

\section{REFERENCES}

1. Blümcke I, Thom M, Aronica E, et al. The clinicopathologic spectrum of focal cortical dysplasias: a consensus classification proposed by an ad hoc Task Force of the ILAE Diagnostic Methods Commission. Epilepsia 2011;52:158-74 CrossRef Medline

2. Taylor DC, Falconer MA, Bruton CJ, et al. Focal dysplasia of the cerebral cortex in epilepsy. J Neurol Neurosurg Psychiatry 1971;34: 369-87 CrossRef Medline

3. Lerner JT, Salamon N, Hauptman JS, et al. Assessment and surgical outcomes for mild type I and severe type II cortical dysplasia: a critical review and the UCLA experience. Epilepsia 2009;50:1310-35 CrossRef Medline

4. Tassi L, Colombo N, Garbelli R, et al. Focal cortical dysplasia: neuropathological subtypes, EEG, neuroimaging and surgical outcome. Brain 2002;125:1719-32 CrossRef Medline

5. Lawson JA, Birchansky S, Pacheco E, et al. Distinct clinicopathologic subtypes of cortical dysplasia of Taylor. Neurology 2005;64:55-61 CrossRef Medline

6. Chassoux F, Landré E, Mellerio C, et al. Type II focal cortical dysplasia: electroclinical phenotype and surgical outcome related to imaging. Epilepsia 2012;53:349-58 CrossRef Medline

7. Guerrini R, Duchowny M, Jayakar P, et al. Diagnostic methods and treatment options for focal cortical dysplasia. Epilepsia 2015;56: 1669-86 CrossRef Medline

8. Colombo N, Tassi L, Deleo F, et al. Focal cortical dysplasia type IIa AJNR Am J Neuroradiol 40:184-90 Jan 2019 www.ajnr.org 
and IIb: MRI aspects in 118 cases proven by histopathology. Neuroradiology 2012;54:1065-77 CrossRef Medline

9. Krsek P, Maton B, Korman B, et al. Different features of histopathological subtypes of pediatric focal cortical dysplasia. Ann Neurol 2008;63:758-69 CrossRef Medline

10. Krsek P, Pieper T, Karlmeier A, et al. Different presurgical characteristics and seizure outcomes in children with focal cortical dysplasia type I or II. Epilepsia 2009;50:125-37 CrossRef Medline

11. Bernasconi A, Antel SB, Collins DL, et al. Texture analysis and morphological processing of magnetic resonance imaging assist detection of focal cortical dysplasia in extra-temporal partial epilepsy. Ann Neurol 2001;49:770-75 CrossRef Medline

12. Focke NK, Symms MR, Burdett JL, et al. Voxel-based analysis of whole brain FLAIR at 3T detects focal cortical dysplasia. Epilepsia 2008;49:786-93 CrossRef Medline

13. Wagner J, Weber B, Urbach H, et al. Morphometric MRI analysis improves detection of focal cortical dysplasia type II. Brain 2011; 134:2844-54 CrossRef Medline

14. Huppertz HJ, Grimm C, Fauser S, et al. Enhanced visualization of blurred gray-white matter junctions in focal cortical dysplasia by voxel-based 3D MRI analysis. Epilepsy Res 2005;67:35-50 CrossRef Medline

15. Wang ZI, Jones SE, Jaisani Z, et al. Voxel-based morphometric magnetic resonance imaging (MRI) postprocessing in MRI-negative epilepsies. Ann Neurol 2015;77:1060-75 CrossRef Medline

16. Huppertz HJ. Morphometric MRI analysis. In: Urbach H, ed. MRI in Epilepsy. Berlin: Springer-Verlag; 2013:73-84

17. Hu WH, Wang X, Liu LN, et al. Multimodality image post-processing in detection of extratemporal MRI-negative cortical dysplasia. Front Neurol 2018;9:450 CrossRef Medline

18. Besson P, Andermann F, Dubeau F, et al. Small focal cortical dysplasia lesions are located at the bottom of a deep sulcus. Brain 2008;131: 3246-55 CrossRef Medline

19. Wang ZI, Alexopoulos AV, Jones SE, et al. Linking MRI postprocessing with magnetic source imaging in MRI-negative epilepsy. Ann Neurol 2014;75:759-70 CrossRef Medline

20. Wellmer J, Parpaley $Y$, von Lehe M, et al. Integrating magnetic resonance imaging postprocessing results into neuronavigation for electrode implantation and resection of subtle focal cortical dysplasia in previously cryptogenic epilepsy. Neurosurgery 2010;66:18794; discussion 194-95 CrossRef Medline

21. Engel JJ, Van Ness PC, Rasmussen TB, et al. Outcome with respect to epileptic seizures. In: Engel JJ, ed. Surgical Treatment of the Epilepsies. 2nd ed. New York: Raven Press; 1993:609-21

22. Barkovich AJ, Kuzniecky RI, Jackson GD, et al. A developmental and genetic classification for malformations of cortical development. Neurology 2005;65:1873-87 CrossRef Medline

23. Marusic P, Najm IM, Ying Z, et al. Focal cortical dysplasias in eloquent cortex: functional characteristics and correlation with MRI and histopathologic changes. Epilepsia 2002;43:27-32 Medline

24. Urbach H, Scheffler B, Heinrichsmeier T, et al. Focal cortical dysplasia of Taylor's balloon cell type: a clinicopathological entity with characteristic neuroimaging and histopathological features, and favorable postsurgical outcome. Epilepsia 2002;43:33-40 Medline

25. Noe K, Sulc V, Wong-Kisiel L, et al. Long-term outcomes after nonlesional extratemporal lobe epilepsy surgery. JAMA Neurol 2013;70: 1003-08 CrossRef Medline

26. Téllez-Zenteno JF, Hernández Ronquillo L, Moien-Afshari F, et al. Surgical outcomes in lesional and non-lesional epilepsy: a systematic review and meta-analysis. Epilepsy Res 2010;89:310-18 CrossRef Medline

27. Richman DP, Stewart RM, Hutchinson JW, et al. Mechanical model of brain convolutional development. Science 1975;189:18-21 CrossRef Medline

28. Budday S, Steinmann P, Kuhl E. Physical biology of human brain development. Front Cell Neurosci 2015;9:257 CrossRef Medline

29. Van Essen DC. A tension-based theory of morphogenesis and compact wiring in the central nervous system. Nature 1997;385:313-18 CrossRef Medline

30. Diehl B, Tkach J, Piao Z, et al. Diffusion tensor imaging in patients with focal epilepsy due to cortical dysplasia in the temporo-occipital region: electro-clinico-pathological correlations. Epilepsy Res 2010;90:178-87 CrossRef Medline

31. Lee SK, Kim DI, Mori S, et al. Diffusion tensor MRI visualizes decreased subcortical fiber connectivity in focal cortical dysplasia. Neuroimage 2004;22:1826-29 CrossRef Medline

32. Widjaja E, Zarei Mahmoodabadi S, Otsubo H, et al. Subcortical alterations in tissue microstructure adjacent to focal cortical dysplasia: detection at diffusion-tensor MR imaging by using magnetoencephalographic dipole cluster localization. Radiology 2009; 251:206-15 CrossRef Medline

33. Najm IM, Bingaman WE, Luders HO. The use of subdural grids in the management of focal malformations due to abnormal cortical development. Neurosurg Clin N Am 2002;13:87-92, viii-ix Medline 